of Rheumatology, Chicago, United States of America; ${ }^{5}$ Northwestern University, Department of Medical Social Sciences, Chicago, United States of America

Background: Patients undergoing total knee replacement (TKR) are at increased risk of persistent opioid use and dependence

Objectives: To identify patients with persistent high-dose opioid use after TKR using group-based trajectory models (GBTM) and determine predictors of persistent high-dose opioid users using pre-TKR patient characteristics

Methods: Using US Medicare claims (2010-2014), we identified patients aged $\geq 65$ years who underwent a TKR and had no history of cancer or high-dose opioid use (>25 mean morphine equivalents (MME)/day) in the year prior. All patients were continuously enrolled in Medicare for $\geq 360$ days prior to and $\geq 30$ days after the TKR. To determine opioid filling patterns after the surgery, patients were followed up to 360 days from the day of TKR. We modeled 12 monthly indicators of opioid prescription fills as a continuous (MME/day) variable using a censored normal GBTM and categorized patients into 4 groups. The primary outcome was persistent high-dose opioid use defined as patients in trajectory Group 3 (38.8 MME/day) or Group 4 (22.4 MME/day). We split the data into training (2010-2013 data) and test (2014 data) sets and used logistic regression to predict high-dose opioid use vs low-dose opioid use (Groups 1 and 2) as a binary outcome utilizing pre-TKR patient characteristics as candidate predictors using the least absolute shrinkage and selection operator (LASSO) regression for variable selection. A reduced model with only 10 pre-specified variables readily available for clinical use was also considered

Results: The final study cohort included 142,089 patients. The GBTM identified 4 distinct trajectories (Group 1- Short-term, low-dose, Group 2- long-term, low-dose, Group 3- medium-term, high-dose, Group 4-long-term, high-dose) of opioid use in the year after TKR (Figure). Using logistic regression and LASSO, we predicted the probability of persistent high-dose opioid use $(\mathrm{N}=17,171)$ (vs. low-dose opioid use) in the training set $(\mathrm{N}=101,810)$ for an $A \cup C=0.80$. The AUC in the test set $(\mathrm{N}=40,279)$ predicting high opioid use $(\mathrm{N}=5,893)$ was 0.77 . The final model selected 33 variables and identified baseline history of opioid use as the strongest positive predictor of high-dose persistent opioid use. The reduced model with only ten predictors also performed equally well (AUC=0.77) (Table).

Conclusion: In this cohort of older patients with no history of cancer or highdose opioid use at baseline, $16.2 \%$ became high dose (28.1 MME/day) opioid users during the year after TKR. Our prediction model with 10 readily available clinical factors may help identify patients at high risk of future adverse outcomes from persistent opioid use and dependence after TKR

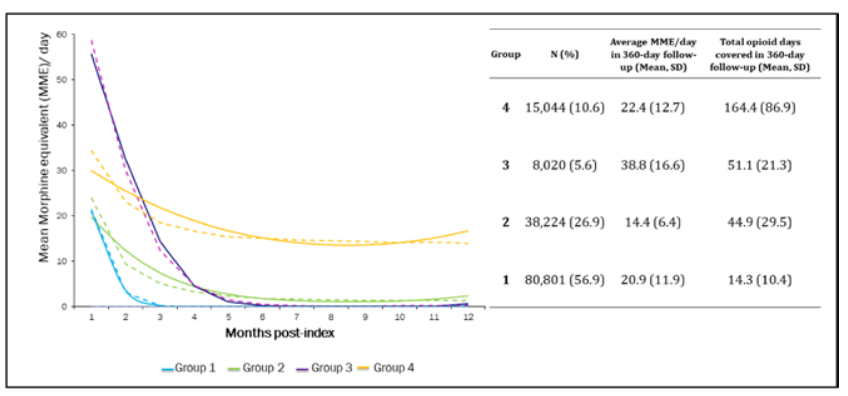

Figure. Trajectories of opioid use patterns after TKR

Table. Predictors of persistent high-dose opioid use in the reduced model

\begin{tabular}{lcc}
\hline Variable & $\begin{array}{c}\text { Multivariable Odds Ratio }(95 \% \mathrm{Cl}) \\
\text { Predicting High dose vs. } \\
\text { Low dose opioid use }\end{array}$ & P-value \\
\hline Age (in years) & $0.94(0.93-0.94)$ & $<0.001$ \\
Females (Ref=Males) & $0.99(0.93-1.06)$ & 0.78 \\
White race (Ref=Other) & $1.25(1.04-1.50)$ & 0.02 \\
Baseline opioid use (MME/day) & $1.22(1.22-1.23)$ & $<0.001$ \\
Substance use (Yes/No) & $1.10(1.02-1.20)$ & 0.02 \\
Benzodiazepine use (Yes/No) & $1.22(1.12-1.32)$ & $<0.001$ \\
Anxiolytic use (Yes/No) & $1.30(1.19-1.43)$ & 0.001 \\
Anticonvulsant use (Yes/No) & $0.94(0.87-1.03)$ & 0.19 \\
Antidepressant use (Yes/No) & $1.03(0.96-1.11)$ & 0.04 \\
NSAID use (Yes/No) & $1.07(1.00-1.14)$ & \\
\hline
\end{tabular}

Disclosure of Interests: Chandrasekar Gopalakrishnan: None declared, Jessica Franklin: None declared, Yinzhu Jin: None declared, Daniel Solomon Grant/ research support from: Funding from Abbvie and Amgen unrelated to this work, Jeffrey Katz Grant/research support from: Dr Katz reported receiving grants from Samumed and Flexion Therapeutics outside the submitted work., Yvonne Lee
Shareholder of: Cigna-Express Scripts, Grant/research support from: Pfizer, Consultant of: Highland Instruments, Inc., Patricia Franklin: None declared, Joyce Lii: None declared, Rishi J Desai Grant/research support from: Dr. Desai reported receiving grants from Bayer, Novartis, and Vertex Pharmaceuticals outside the submitted work., Seoyoung Kim Grant/research support from: Seoyoung $\mathrm{C}$ Kim has received research grants from AbbVie, Roche, Bristol-Myers Squibb and Pfizer.

DOI: 10.1136/annrheumdis-2020-eular.4017

\section{OP0072 \\ IDENTIFYING POLYMYALGIA RHEUMATICA RELAPSE AND ITS ASSOCIATIONS IN A RETROSPECTIVE COHORT}

T. Bolhuis ${ }^{1}$, D. Marsman ${ }^{1}$, N. Den Broeder ${ }^{1}$, F. Van den Hoogen ${ }^{1,2}$, A. Den Broeder ${ }^{1,2}$, A. Van der Maas'. ' 'Sint Maartenskliniek, Rheumatology, Nijmegen, Netherlands; ${ }^{2}$ Radboudumc, Rheumatology, Nijmegen, Netherlands

Background: Polymyalgia rheumatica (PMR) relapse occurs frequently when tapering glucocorticoids $(\mathrm{GC})^{1}$. Some risk factors for varying definitions of relapse have been identified, although with conflicting results ${ }^{2}$. Identification of relapse and its associations can help identify patients in need of tighter follow up or additional medication.

Objectives: To identify PMR GC-taper related relapse (proportion 1 and 2 years after starting treatment and per year of treatment) and candidate predictors, for a future prediction model.

Methods: In a retrospective cohort of new PMR patients, visiting our hospital from April 2008 - January 2018, all visits $>30$ days after starting GC treatment and with $>2.5 \mathrm{mg}$ oral prednisolone were used to identify substantial relapses. Relapse was defined in two ways: rheumatologist judgement (RJ) and treatment intensivation ( $\mathrm{TI})$. Agreement between $\mathrm{RJ}$ and $\mathrm{TI}$ at visits was assessed. TI relapse was used going forward for treatment based prediction. The proportion of relapsers after 1 and 2 years (cumulative incidence) and the amount of relapses per year of treatment (incidence rate (IR)), were assessed. Unadjusted associations with candidate predictors, present when starting GC treatment, were assessed using logistic and Poisson regression respectively.

Results: Data from 417 patients was used (figure 1). Relapse occurred at 405 and 325 (of 2455) visits based on RJ and TI respectively. TI relapse (cumulative incidence) after 1 and 2 years was $134(32 \%)$ and 184 (44\%) and IR was 0.35 per patient year. Unadjusted significant associations for the cumulative incidence were CRP and ESR at baseline, and symptom duration before treatment (table 1), but only CRP and ESR were significantly associated with yearly IR.

Table 1: Associations between predictors and relapse after 1 and 2 years of treatment and the amount of relapses per year of treatment.

\begin{tabular}{|c|c|c|c|c|c|c|c|c|c|}
\hline \multirow{3}{*}{$\begin{array}{l}\text { Predictors } \\
\text { Age - years }\end{array}$} & \multicolumn{3}{|c|}{ Within first year } & \multicolumn{3}{|c|}{ Within first 2 years } & \multicolumn{3}{|c|}{ Incidence rate } \\
\hline & \multirow{2}{*}{$\frac{\mathrm{OR}}{1.00}$} & \multicolumn{2}{|c|}{$\mathrm{Cl}$} & \multirow{2}{*}{$\begin{array}{l}\text { OR } \\
1.00\end{array}$} & \multicolumn{2}{|c|}{$\mathrm{Cl}$} & \multirow{2}{*}{$\begin{array}{l}\text { IRR } \\
1.00\end{array}$} & \multicolumn{2}{|c|}{$\mathrm{Cl}$} \\
\hline & & 0.98 & 1.03 & & 0.98 & 1.02 & & 0.99 & 1.01 \\
\hline Sex - ref. Male & 1.44 & 0.95 & 2.20 & 1.38 & 0.94 & 2.05 & 1.38 & 0.79 & 1.27 \\
\hline \multicolumn{10}{|l|}{ Medical history - ref. No } \\
\hline - inflammatory & 1.18 & 0.75 & 1.86 & 1.00 & 0.65 & 1.53 & 1.00 & 0.95 & 1.60 \\
\hline - malignancy & 0.69 & 0.36 & 1.35 & 0.96 & 0.53 & 1.73 & 0.96 & 0.64 & 1.35 \\
\hline - cardiovascular & 1.68 & 0.07 & 2.95 & 1.74 & 1.00 & 3.03 & 1.74 & 0.89 & 1.66 \\
\hline \multicolumn{10}{|l|}{ Smoking - ref. no, $n=336$} \\
\hline Stopped & 0.85 & 0.49 & 1.46 & 1.16 & 0.70 & 1.92 & 1.16 & 0.96 & 1.74 \\
\hline Yes & 0.81 & 0.412 & 1.57 & 0.98 & 0.53 & 1.82 & 0.98 & 0.67 & 1.40 \\
\hline $\begin{array}{l}\text { Symptom duration before } \\
\text { baseline }- \text { per week, } n=411\end{array}$ & 0.98 & 0.96 & 0.998 & 0.98 & 0.964 & 0.997 & 0.98 & 0.98 & 1.00 \\
\hline $\begin{array}{l}\text { Clinical disease severity - } \\
\text { score } 0-8\end{array}$ & 0.93 & 0.82 & 1.07 & 0.93 & 0.82 & 1.05 & 0.93 & 0.87 & 1.02 \\
\hline $\begin{array}{l}\text { (Suspected) presence of } \\
\quad \text { peripheral arthritis - ref. No }\end{array}$ & 0.73 & 0.40 & 1.33 & 0.67 & 0.38 & 1.16 & 0.67 & 0.55 & 1.16 \\
\hline $\begin{array}{l}\text { Presence of systemic } \\
\text { symptoms - ref. No }\end{array}$ & 0.78 & 0.51 & 1.19 & 0.84 & 0.57 & 1.25 & 0.84 & 0.79 & 1.28 \\
\hline CRP - per mg/L, $n=363$ & 1.01 & 1.00 & 1.01 & 1.01 & 1.00 & 1.01 & 1.01 & 1.00 & 1.01 \\
\hline ESR - per $\mathrm{mm} / \mathrm{h}, \mathrm{n}=396$ & 1.02 & 1.01 & 1.03 & 1.02 & 1.01 & 1.03 & 1.02 & 1.00 & 1.01 \\
\hline $\mathrm{Hb}-$ per $\mathrm{mmol} / \mathrm{L}, \mathrm{n}=316$ & 1.03 & 0.75 & 1.39 & 0.99 & 0.74 & 1.32 & 0.99 & 0.86 & 1.25 \\
\hline APR - ref. normal, $n=376$ & 0.99 & 0.41 & 2.40 & 0.79 & 0.34 & 1.83 & 0.79 & 0.63 & 1.68 \\
\hline
\end{tabular}

Ref., reference category; $\mathrm{Hb}$, haemoglobin; APR, acute phase reactants; $\mathrm{Cl}, 95 \%$ confidence interval

Conclusion: PMR relapse while tapering GC occurs frequently, and some - although weak - associations were found. Longer symptom duration before treatment decreased chance of relapse, but did not increase the amount of relapses per year of treatment, potentially indicating a more self-limiting disease course. A uniform definition of relapse and identifying further predictors for a potential prediction model is needed to focus GC sparing agents for patients. 
References:

[1] Mackie SL et al. Can the prognosis of polymyalgia rheumatica be predicted at disease onset? Results from a 5-year prospective study. Rheumatology (Oxford). 2010;49(4):716-22.

[2] Dejaco $\mathrm{C}$ et al. Current evidence for therapeutic interventions and prognostic factors in polymyalgia rheumatica: A systematic literature review informing the 2015 EULAR/ACR. ARD. 2015;74: 1808-17.

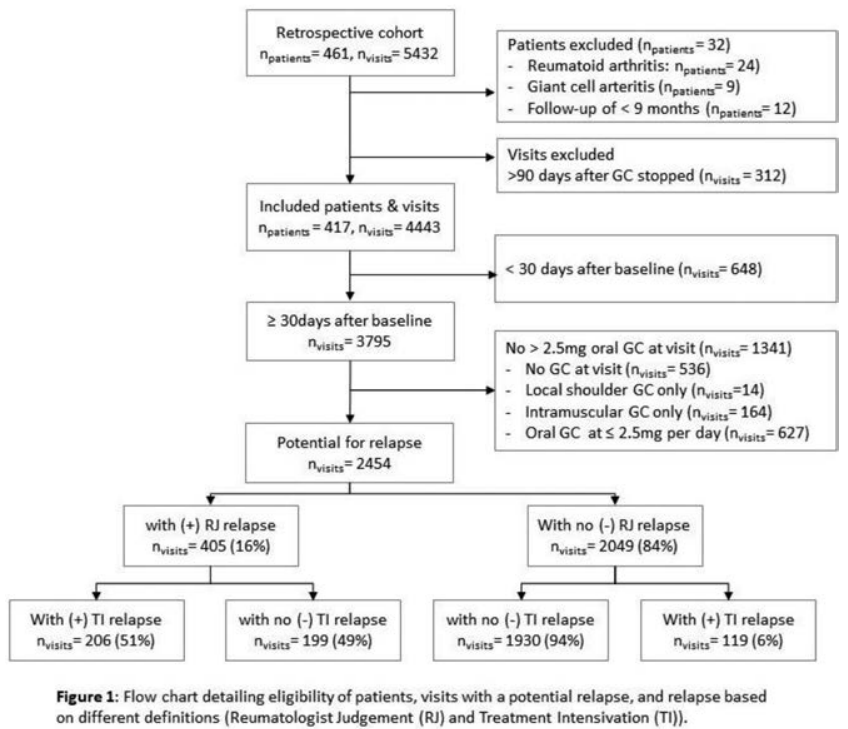

Disclosure of Interests: None declared

DOI: 10.1136/annrheumdis-2020-eular.3560

\section{OP0073 $\quad$ RISK OF DEVELOPING RHEUMATIC DISEASES IN PATIENTS WITH PALINDROMIC RHEUMATISM IN SOUTH KOREA: A NATION-WIDE POPULATION- BASED STUDY}

J. K. Ahn ${ }^{1}$, J. Hwang ${ }^{2}$, J. Lee ${ }^{3}$, H. Kim ${ }^{3}$, G. H. Seo ${ }^{4} .{ }^{1}$ Kangbuk Samsung Hospital, Sungkyunkwan University School of Medicine, Division of Rheumatology, Department of Internal Medicine, Seoul, Korea, Rep. of (South Korea); ${ }^{2}$ Samsung Changwon Hospital, Sungkyunkwan University School of Medicine, Division of Rheumatology, Department of Internal Medicine, Changwon-si, Korea, Rep. of (South Korea); ${ }^{3}$ Samsung Medical Center, Sungkyunkwan School of Medicine, Department of Medicine, Seoul, Korea, Rep. of (South Korea); ${ }^{4}$ Health Insurance Review and Assessment Service, Wonju, Korea, Rep. of (South Korea)

Background: Palindromic rheumatism (PR) has known to be three patterns of disease course: clinical remission of attacks, persistent attacks, and evolution to chronic arthritis or systemic disease. The spectrum in progression to chronic diseases of PR, however, is quite variable; rheumatoid arthritis (RA), systemic lupus erythematosus (SLE), systemic sclerosis (SSc), Sjögren's syndrome (SjS), ankylosing spondylitis (AS), relapsing polychondritis (RP), Behçet's disease (BD), sarcoidosis, and psoriatic spondylitis and arthropathy. Because of the small numbers in case-control studies and too aged investigations, now we needs to shed new light on the fate of PR.

Objectives: The aim was to investigate the epidemiology of PR and the risk of developing various rheumatic diseases compared with non-PR individuals, employing the National Health Insurance Service (NHIS) medical claims data, which covers all medical institutions of South Korea.

Methods: The study used 2007-2018 claims data from the Korean Health Insurance Review and Assessment Service (HIRA). The identified 19,724 PR patients from 2010 to 2016 were assessed for the incidence rate (IR) compared with the population in the given year by 100,000 person-year (py). The date of diagnosis was the index date. After matching with non-PR individuals (1:10) for age, sex and the year of index date, we calculated the hazard ratios (HRs) with 95\% confidence intervals (Cls). The risk of developing the various rheumatic diseases and adult immunodeficiency syndrome (AIDS) as the outcome diseases in PR cohort was estimated. This risk was compared with that of matched non-PR cohort.

Results: Of 19,724 PR patients (8,665 males and 11,059 females), the mean age was $50.2 \pm 14.9$ years $(47.7 \pm 14.4$ years in males and $52.6 \pm 14.9$ years in females, $p<0.001)$. The ratio of male to female patients with $\mathrm{PR}$ was approximately $1: 1.28$. The annual IR of PR was $7.02(6.92-7.12)$ per 100,000 py (6.22 (6.09-6.35) and $7.80(7.66-7.95)$ per 100,000 py in males and females, respectively). The mean duration to develop the outcome diseases was significantly shorter in PR cohort compared that of non-PR cohort (19.4 vs. 35.8 months, $p<0.001)$. The most common outcome disease was RA $(7.34 \%$ of PR patients; $80.0 \%$ of total outcome diseases), followed by AS, SLE, BD, SjS MCTD, DM/PM, SSc, RP, psoriatic arthropathy, and AIDS in PR cohort. The patients with PR had an increased risk of RA (HR 46.6, 95\% Cl [41.1-52.7]) psoriatic arthropathy (44.79 [15.2-132.4]), SLE (24.5 [16.2-37.2]), MCTD (22.0 [7.7-63.3]), BD (21.0 [13.8-32.1]), SjS (12.4 [8.5-17.9]), AS (9.0 [6.7-12.2]), DM/ PM (6.1 [2.6-14.8]), and SSc (3.8 [1.5-9.6]) but not of AIDS. The risk of developing RA was greater in male patients (HR 58.9, 95\% Cl [45.6-76.2] vs. 43.2 [37.4-49.8], $p$ for interaction $=0.037$ ) while female patients encountered a higher risk of developing AS (15.8 [8.9-28.1] vs. 7.2 [5.0-10.3], $p$ for interaction $=0.023$ ). The risk of developing RA, SLE, SjS, and BD were significantly more highly affected in younger age $(p$ for interaction $<0.001,=0.003,0.002$, and 0.017 , at each).

Conclusion: This nationwide, population-based cohort study demonstrated that patients with PR had an increased risk of developing various rheumatic diseases, not only RA but also psoriatic arthropathy. Therefore, patients with PR needs to be cautiously followed up for their potential of diverse outcome other than RA: RA, SLE, SjS, and BD in younger patients, RA in males, and AS in females, in particular.

Disclosure of Interests: None declared

DOI: 10.1136/annrheumdis-2020-eular.3664

\section{OP0074 MULTIMORBIDITY CLUSTERS, DETERMINANTS AND TRAJECTORIES IN OSTEOARTHRITIS IN THE UK: FINDINGS FROM THE CLINICAL PRACTICE RESEARCH DATALINK}

S. Swain ${ }^{1}$, C. Coupland ${ }^{2}$, V. Strauss ${ }^{3}$, C. Mallen ${ }^{4}$, C. F. Kuo ${ }^{5}$, A. Sarmanova ${ }^{6}$, M. Doherty ${ }^{1}$, W. Zhang ${ }^{1}{ }^{1}$ University of Nottingham, Academic Rheumatology, Nottingham, United Kingdom; ${ }^{2}$ University of Nottingham, Division of Primary Care, Nottingham, United Kingdom; ${ }^{3}$ University of Oxford, NDORMS, Oxford, United Kingdom; ${ }^{4}$ Keele University, School of Primary, Community and Social Care, Keele, United Kingdom; ${ }^{5}$ Chang Gung Memorial Hospital, Division of Rheumatology, Allergy and Immunology, Taiwan, Taiwan, Republic of China; ${ }^{6}$ University of Bristol, Musculoskeletal Research Unit, Bristol Medical School, Bristol, United Kingdom

Background: Multimorbidity ( $\geq 2$ chronic conditions) escalates the risk of adverse health outcomes. However, its burden in people with osteoarthritis (OA) remains largely unknown.

Objectives: To identify the clusters of patients with multimorbidity and associated factors in OA and non-OA populations and to estimate the risk of developing multimorbidity clusters after the index date (after diagnosis).

Methods: The study used the Clinical Practice Research Datalink - a primary care database from the UK. Firstly, age, sex and practice matched OA and non-OA people aged 20+ were identified to explore patterns and associations of clusters of multimorbidity within each group. Non-OA controls were assigned with same index date as that of matched OA cases. Secondly, multimorbidity trajectories for 20 years after the index date were examined in people without any comorbidities at baseline in both $\mathrm{OA}$ and non-OA groups. Latent class analysis was used to identify clusters and latent class growth modelling was used for cluster trajectories. The associations between clusters and age, sex, body mass index (BMI), alcohol use, smoking habits at baseline were quantified through multinomial logistic regression.

Results: In total, 47 long-term conditions were studied in 443,822 people (OA221922; non-OA- 221900), with a mean age of 62 years (standard deviation \pm 13 years), and $58 \%$ being women. The prevalence of multimorbidity was $76.6 \%$ and $68.9 \%$ in the OA and non-OA groups, respectively. In the OA group five clusters were identified including relatively healthy (18\%), "cardiovascular (CVD) and musculoskeletal (MSK)' (12.3\%), metabolic syndrome (28.2\%), 'pain and psychological (9.1\%), and 'musculoskeletal' (32.4\%). The non-OA group had similar patterns except that the 'pain+ psychological' cluster was replaced by 'thyroid and psychological'. (Figure 1) Among people with OA, 'CVD+MSK' and metabolic syndrome clusters were strongly associated with obesity with a relative risk ratio (RRR) of $2.04(95 \% \mathrm{Cl} 1.95-2.13)$ and $2.10(95 \% \mathrm{Cl} 2.03-2.17)$, respectively Women had four times higher risk of being in the 'pain+ psychological' cluster than men when compared to the gender ratio in the healthy cluster, (RRR 4.28; $95 \% \mathrm{Cl} 4.09-4.48)$. In the non-OA group, obesity was significantly associated with all the clusters. 